\title{
CK20 versus AMACR and p53 immunostains in evaluation of Urothelial Carcinoma in Situ and Reactive Atypia
}

\author{
Daniel J. Neal ${ }^{1}$, Mahul B. Amin ${ }^{2}$ and Steven C. Smith ${ }^{1,3^{*}}$ (D)
}

\begin{abstract}
Ancillary testing with immunohistochemistry has shown recent promise in the workup of equivocal bladder lesions. We read with interest the recent findings of Alston et al., who assessed the diagnostic utility of alpha-methylacylCoA racemase (AMACR) in comparison to cytokeratin 20 (CK20) in evaluation of atypia in challenging flat urothelial lesions in the differential between carcinoma in situ (CIS) and reactive atypia. AMACR was reported to be a somewhat more specific but less sensitive marker for CIS than CK20, though showing weaker intensity. Spurred by their report, with the knowledge that we had consistently and consecutively performed AMACR, CK20, and p53 on flat urothelial lesions challenging enough to reach intradepartmental consensus, we performed a retrospective review. Similarly, we found that AMACR was less sensitive (80\%) and more specific (100\%) than CK20, with the same caveat of less staining intensity. Additionally, our p53 review identified a significant rate $(\sim 27 \%)$ of equivocal/noninformative findings. Taken together, our experience in this consecutive cohort confirms the impression of Alston et al. regarding the utility and challenges of AMACR use, while highlighting challenges with p53, which we plan to use more sparingly prospectively.
\end{abstract}

Keywords: Urothelial carcinoma in situ, Cytokeratin 20, Alpha-methylacyl-CoA racemase

\section{Introduction}

Distinguishing between urothelial carcinoma in situ (CIS) and reactive cellular atypia in bladder specimens can prove challenging $[1,2]$, requiring careful correlation with clinical and cytologic features $[3,4]$. Though recent studies have shown the overexpression of cytokeratin 20 (CK20), p53 and even alpha-methylacyl-CoA racemase (AMACR) in CIS [5-7], few studies have evaluated each marker's performance in consecutive clinical cases. Recently Alston et al. reviewed 52 cases in which immunohistochemistry (IHC) for CK20 and AMACR was performed on cases with final diagnoses consisting of

\footnotetext{
*Correspondence: steven.c.smith@vcuhealth.org

'Department of Pathology, Virginia Commonwealth University School of Medicine, PO Box 980662, Richmond, VA 23298, USA

${ }^{3}$ Division of Urology, Department of Surgery, Virginia Commonwealth University School of Medicine, PO Box 980662, Richmond, VA 23298, USA

Full list of author information is available at the end of the article
}

CIS or non-neoplastic/reactive [8]. They additionally identified and stained 20 unequivocal cases of CIS in which prior IHC was not performed. In their experience, AMACR had a sensitivity of $73 \%$ and specificity of $97 \%$, while CK20 had a sensitivity of $95 \%$ and specificity of $80 \%$. CK20 showed stronger, more consistent staining, while weak, surface positivity was seen in both among non-neoplastic cases. Based on its higher sensitivity and consistent, strong staining pattern, CK20 was suggested as perhaps a better suited ancillary test for distinguishing CIS versus reactive atypia. Given that in our department we had as a standard procedure ordered AMACR and CK20, as well as p53, on cases thought to require consensus opinion in recent years, we performed herein a retrospective review for comparison. 
Table 1 CK20, AMACR, and p53 staining in Intradepartmental Consensus Urothelial Lesions

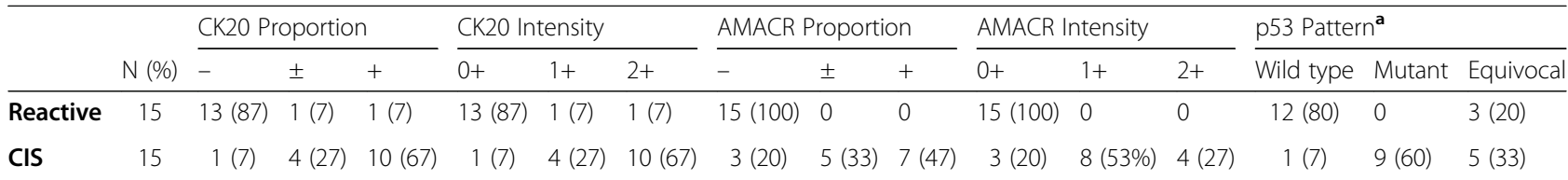

Abbreviations: CK20 cytokeratin 20, AMACR alpha-methylacyl-CoA racemase;

Staining Proportion: -, negative; \pm , patchy; + , diffuse

Staining Intensity: $0+$ negative; $1+$ weak; $2+$ strong

a Please see Methods for description of scoring pattern

\section{Methods}

We retrospectively reviewed 31 cases from 29 different patients from 2014 to 2019 (CK20 clone SP33; AMACR clone SP116; p53 clone DO-7; all predilute and performed on a Ventana Benchmark XT). All slides were re-reviewed, including original $\mathrm{H} \& \mathrm{E}$ and $\mathrm{IHC}$ stains, for confirmation of diagnoses and exclusion of processes such as atypia related to BK virus [9]. Clinicopathologic features were tabulated. Staining for CK20 and AMACR was evaluated similarly to Alston et al. [8] as negative
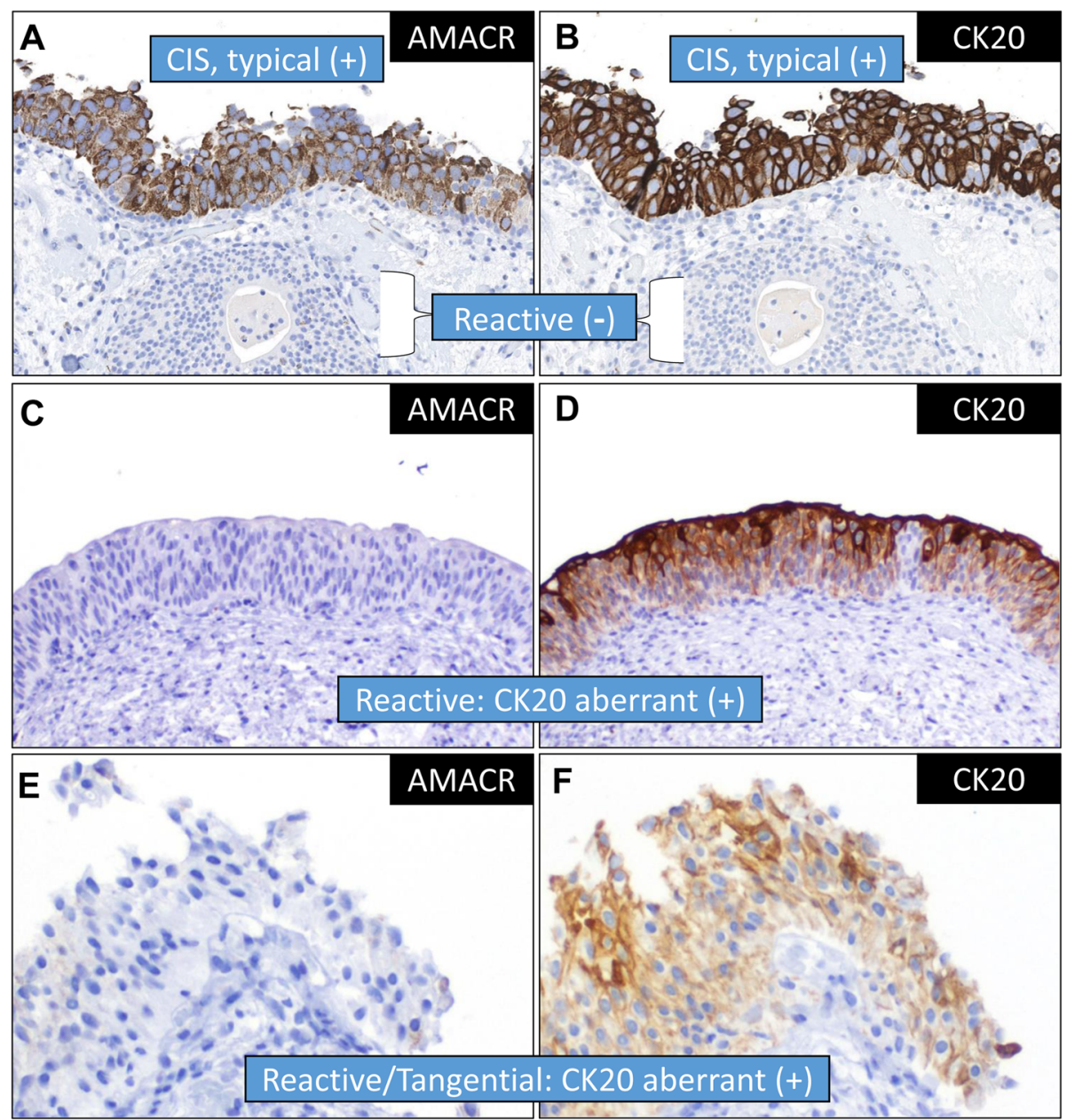

Fig. 1 The most characteristic pattern of staining in urothelial carcinoma in situ (CIS) was of diffuse, full thickness (+) staining for AMACR (a) and CK20 (b). AMACR typically demonstrated reduced intensity and a granular cytoplasmic pattern, as compared to CK20; additionally, reactive urothelium in the nest of cystitis cystica underlying the surface CIS demonstrates expected negativity for both markers. Two examples of the challenges in the use of these markers where AMACR added utility included one case of reactive urothelium showing negative AMACR (c) but aberrantly positive CK20, relatively strong in most of the thickness of the urothelium (d). Additionally, we noted examples of small biopsies where AMACR was negative (e) but CK20 appeared positive (f), even if relatively weakly so, due in part to staining of normal umbrella cells sectioned tangentially en face 
(focal/luminal expression), patchy (partial positive $>1 / 3$ but $\leq 2 / 3$ of the urothelium), or diffuse ( $>2 / 3$ thickness), with intensity scored as negative $(0)$, weak $(1+)$, or strong (2+). Evaluation of nuclear p53 expression was scored as wild-type (scattered, usually weak-moderate) mutant-type (strong consistent staining or complete absence of staining in atypical cells) or equivocal (any other pattern observed).

\section{Results}

A total of 31 samples from 29 patients (17 M; 12 F) were reviewed, with median age of 65 years. Specimens including 16 bladder biopsies, 11 transurethral resections (TURBTs), 2 resections including one partial cystectomy and one pelvic exenteration, 1 ureter biopsy, and one transurethral resection of the prostate (TURP). Final diagnoses included 15 CIS and 15 reactive urothelium (Table 1), with an additional case finally diagnosed as high-grade squamous intraepithelial lesion (HSIL) of gynecologic origin. Of cases with a final diagnosis of CIS, 14 of 15 (93\%) showed positive staining for CK20, compared to 12 of $15(80 \%)$ positive with AMACR. However, of cases diagnosed as reactive atypia, 2 of 15 (13\%) showed positive staining for CK20, compared to 0 of 15 (0\%) for AMACR. As expected, CK20 showed greater staining in non-neoplastic umbrella cells. AMACR staining was statistically significantly less intense but not less diffuse than CK20 ( $p=0.02$ and $p=0.07$, respectively, paired Wilcoxon). Across all specimens, p53 showed a significant rate of equivocal/non-informative staining (27\%), disproportionately (63\%) among cases diagnosed as CIS.

\section{Discussion}

In conjunction with the findings from Alston et al., our results support the use of CK20 and AMACR IHC in equivocal urothelial lesions in the differential between CIS and reactive atypia (Fig. 1a-b). We confirm the finding of AMACR showing marginally less sensitivity, more specificity (one example in Fig. 1c-d), but less intensity overall. Anecdotally, we also saw cases with areas of tangential sectioning of umbrella cells simulating CK20 positivity but where AMACR was negative (Fig. 1e-f) due to its essential lack of expression in non-neoplastic urothelium. This may represent one relative advantage of AMACR; in any case, given our significant rate of equivocal findings we plan to substitute AMACR for p53 going forward. While the reasons for CK20 and AMACR expression is CIS remain unknown [10], the addition of AMACR to the diagnostic armamentarium in this setting is welcome.

\section{Abbreviations}

AMACR: alpha-methylacyl-CoA racemase; CK20: cytokeratin 20; IHC: immunohistochemistry

\section{Acknowledgements}

None

\section{Authors' contributions}

DJN and SCS conceptualized the project, collected, analyzed data, wrote the manuscript, and edited and approved the final version. MBA conceptualized the project, analyzed data, and edited and approved the final version.

\section{Funding}

There is no funding source to disclose.

Availability of data and materials

The datasets used and analysed during the current study are available from the corresponding author on reasonable request.

Ethics approval and consent to participate

This study was approved by the Virginia Commonwealth University RAMSIRB under an expedited protocol with waiver of informed consent

Consent for publication

Not applicable

\section{Competing interests}

The authors declare that they have no competing interests.

\section{Author details}

${ }^{1}$ Department of Pathology, Virginia Commonwealth University School of Medicine, PO Box 980662, Richmond, VA 23298, USA. ²Department of Pathology and Laboratory Medicine, University of Tennessee Health Sciences Center, Memphis, TN 38163, USA. ${ }^{3}$ Division of Urology, Department of Surgery, Virginia Commonwealth University School of Medicine, PO Box 980662, Richmond, VA 23298, USA.

Received: 18 February 2020 Accepted: 20 May 2020

Published online: 26 May 2020

References

1. Amin MB, Trpkov K, Lopez-Beltran A, Grignon D, et al. Best practices recommendations in the application of immunohistochemistry in the bladder lesions: report from the International Society of Urologic Pathology consensus conference. Am J Surg Pathol. 2014;38(8):e20-34.

2. McKenney JK, Gomez JA, Desai S, Lee MW, Amin MB. Morphologic expressions of urothelial carcinoma in situ: a detailed evaluation of its histologic patterns with emphasis on carcinoma in situ with microinvasion. Am J Surg Pathol. 2001;25(3):356-62.

3. Hassan M, Solanki S, Kassouf W, Kanber Y, Caglar D, Auger M, et al. Impact of implementing the Paris system for reporting urine cytology in the performance of urine cytology: a correlative study of 124 cases. Am J Clin Pathol. 2016;146(3):384-90.

4. Sassa N, Iwata $H$, Kato M, Murase $Y$, Seko S, Nishikimi T, et al. Diagnostic utility of UroVysion combined with conventional urinary cytology for Urothelial carcinoma of the upper urinary tract. Am J Clin Pathol. 2019; 151(5):469-78.

5. Aron M, Luthringer DJ, Mckenney JK, Hansel DE, Westfall DE, Parakh R, et al. Utility of a triple antibody cocktail intraurothelial neoplasm-3 (IUN-3-CK20/ CD44s/p53) and a-methylacyl-CoA racemase (AMACR) in the distinction of urothelial carcinoma in situ (CIS) and reactive urothelial atypia. Am J Surg Pathol. 2013;37(12):1815-23.

6. Arias-Stella JA 3rd, Shah AB, Gupta NS, Williamson SR. CK20 and p53 Immunohistochemical staining patterns in urinary bladder specimens with equivocal Atypia. Arch Pathol Lab Med. 2018;142(1):64-9.

7. Gunia S, Koch S, Hakenberg OW, May M, Kakies C, Erbersdobler A. Different HER2 protein expression profiles aid in the histologic differential diagnosis between Urothelial carcinoma in situ (CIS) and non-CIS conditions (dysplasia and reactive Atypia) of the urinary bladder mucosa. Am J Clin Pathol. 2011 Jan 12;136(6):881-8.

8. Alston ELJ, Zynger DL. Does the addition of AMACR to CK20 help to diagnose challenging cases of urothelial carcinoma in situ? Diagn Pathol. 2019;14(1):91

9. Chu YH, Zhong W, Rehrauer W, Pavelec DM, Ong IM, Arjang D, et al. Clinicopathologic characterization of post-renal transplantation BK 
Polyomavirus-associated Urothelial carcinoma single institutional experience. Am J Clin Pathol. 2020;153(3):303-14.

10. Langner C, Rupar G, Leibl S, Hutterer G, Chromecki T, Hoefler G, et al. Alphamethylacyl-CoA racemase (AMACR/P504S) protein expression in urothelial carcinoma of the upper urinary tract correlates with tumour progression. Virchows Arch. 2006;448(3):325-30.

\section{Publisher's Note}

Springer Nature remains neutral with regard to jurisdictional claims in published maps and institutional affiliations.

Ready to submit your research? Choose BMC and benefit from:

- fast, convenient online submission

- thorough peer review by experienced researchers in your field

- rapid publication on acceptance

- support for research data, including large and complex data types

- gold Open Access which fosters wider collaboration and increased citations

- maximum visibility for your research: over $100 \mathrm{M}$ website views per year

At $B M C$, research is always in progress.

Learn more biomedcentral.com/submissions 